\title{
Non-damping oscillations at flaring loops ${ }^{\star}$
}

\author{
D. Li (李东) $)^{1,2,3}$, D. Yuan ${ }^{4}$, Y. N. Su${ }^{1,5}$, Q. M. Zhang ${ }^{1,3}$, W. Su${ }^{6}$, and Z. J. Ning ${ }^{1,5}$
}

\footnotetext{
1 Key Laboratory for Dark Matter and Space Science, Purple Mountain Observatory, CAS, Nanjing 210034, PR China e-mail: lidong@pmo.ac.cn, ningzongjun@pmo.ac.cn

2 Sate Key Laboratory of Space Weather, Chinese Academy of Sciences, Beijing 100190, PR China

3 CAS Key Laboratory of Solar Activity, National Astronomical Observatories, Beijing 100012, PR China

${ }^{4}$ Institute of Space Science and Applied Technology, Harbin Institute of Technology, Shenzhen Campus, Shenzhen 518055, PR China

5 School of Astronomy and Space Science, University of Science and Technology of China, Hefei, Anhui 230026, PR China

${ }^{6}$ MOE Key Laboratory of Fundamental Physical Quantities Measurements, School of Physics, Huazhong University of Science and Technology, Wuhan 430074, PR China
}

Received 9 March 2018 / Accepted 9 June 2018

\begin{abstract}
Context. Quasi-periodic oscillations are usually detected as spatial displacements of coronal loops in imaging observations or as periodic shifts of line properties (i.e., Doppler velocity, line width and intensity) in spectroscopic observations. They are often applied for remote diagnostics of magnetic fields and plasma properties on the Sun.

Aims. We combine the imaging and spectroscopic measurements of available space missions, and investigate the properties of nondamping oscillations at flaring loops.

Methods. We used the Interface Region Imaging Spectrograph (IRIS) to measure the spectrum over a narrow slit. The doublecomponent Gaussian fitting method was used to extract the line profile of Fe xxi $1354.08 \AA$ at the "O I" spectral window. The quasiperiodicity of loop oscillations were identified in the Fourier and wavelet spectra.

Results. A periodicity at about $40 \mathrm{~s}$ is detected in the line properties of Fe xxı $1354.08 \AA$, hard X-ray emissions in GOES $1-8 \AA$ derivative, and Fermi $26-50 \mathrm{keV}$. The Doppler velocity and line width oscillate in phase, while a phase shift of about $\pi / 2$ is detected between the Doppler velocity and peak intensity. The amplitudes of Doppler velocity and line width oscillation are about $2.2 \mathrm{~km} \mathrm{~s}^{-1}$ and $1.9 \mathrm{~km} \mathrm{~s}^{-1}$, respectively, while peak intensity oscillates with amplitude at about $3.6 \%$ of the background emission. Meanwhile, a quasi-period of about $155 \mathrm{~s}$ is identified in the Doppler velocity and peak intensity of the Fe xxI $1354.08 \AA$ line emission, and AIA $131 \AA$ A intensity.

Conclusions. The oscillations at about $40 \mathrm{~s}$ are not damped significantly during the observation; this might be linked to the global kink modes of flaring loops. The periodicity at about $155 \mathrm{~s}$ is most likely a signature of recurring downflows after chromospheric evaporation along flaring loops. The magnetic field strengths of the flaring loops are estimated to be about $120-170 \mathrm{G}$ using the magnetohydrodynamic seismology diagnostics, which are consistent with the magnetic field modeling results using the flux rope insertion method.
\end{abstract}

Key words. Sun: flares - Sun: oscillations - Sun: UV radiation - Sun: X-rays, gamma rays - line: profiles - techniques: spectroscopic

\section{Introduction}

Quasi-periodic oscillations are very common phenomena on the Sun. They are detected in a wide range of wavelengths: radio emissions (Ning et al. 2005; Tan \& Tan 2012; Li et al. 2015a), visible lights, extreme-ultraviolet and ultraviolet (EUV and UV) emissions (Aschwanden et al. 2002; De Moortel et al. 2002; Su et al. 2012; Shen et al. 2013; Li et al. 2016a), soft and hard X-ray (SXR and HXR), or even $\gamma$-ray channels (Li \& Gan 2008; Nakariakov et al. 2010; Ning 2014, 2017). They are usually identified as a series of regular and periodic variations in the total emission fluxes (Tan \& Tan 2012; Li \& Gan 2008; Ning 2014; Li et al. 2017a), or the spatial displacements of coronal loops in imaging (Aschwanden et al. 1999; Nakariakov et al. 1999; Shen \& Liu 2012; Shen et al. 2017) or spectroscopic observations (Ofman \& Wang 2002; Wang et al. 2002; Tian et al. 2011, 2012, 2016; Li \& Zhang 2015). The oscillation period in the

\footnotetext{
$\star$ The movie associated to Fig. 1 is available at https: //www . aanda.org
}

same event could be observed only in a single channel (e.g., Ning 2014; Li \& Zhang 2017; Milligan et al. 2017), or simultaneously over a broad wavelength (e.g., Li et al. 2015a; Zhang et al. 2016; Ning 2017). In a few cases, multiple periodicity could be detected in the same event (Inglis \& Nakariakov 2009; Zimovets \& Struminsky 2010; Tian et al. 2016; Yang \& Xiang 2016; Li et al. 2017a; Li \& Zhang 2017; Shen et al. 2018). The detected periods do not form strict harmonics, which might be caused by the expansion of loops (Verth \& Erdélyi 2008), the separation of footpoints (Tian et al. 2016), plasma stratification (Andries et al. 2005), or the siphon flow (Li et al. 2013).

In the past few decades, a variety of quasi-periodic oscillations have been observed in the coronal loops (e.g., Wang et al. 2002; Nakariakov \& Verwichte 2005; De Moortel \& Nakariakov 2012; Zimovets \& Nakariakov 2015; Tian et al. 2016), and are usually interpreted as magnetohydrodynamic (MHD) waves (Nakariakov \& Melnikov 2009; De Moortel \& Nakariakov 2012; Anfinogentov et al. 2015), that is, slow waves (Ofman \& Wang 2002; Wang et al. 2009; Mandal et al. 2016), sausage waves 
(Gruszecki et al. 2012; Tian et al. 2016), and kink waves (Tian et al. 2012; Kumar et al. 2016; Yuan et al. 2016; Li et al. 2017b). These MHD oscillations could be identified in the EUV and SXR imaging observations (Nakariakov et al. 1999; Aschwanden et al. 2002; Shen \& Liu 2012; Shen et al. 2013; Goddard \& Nakariakov 2016), and in the spectroscopic observations (Kliem et al. 2002; Mariska 2005; Li et al. 2015a). The detected periods vary from a few seconds to minutes (Aschwanden et al. 2002; Schrijver et al. 2002; Tian et al. 2016; Verwichte \& Kohutova 2017).

Kink oscillations of coronal loops are the most commonlymeasured modes (Nakariakov \& Verwichte 2005; Ruderman \& Erdélyi 2009; Nakariakov et al. 2016). They are often detected as transverse displacements of coronal loops in the imaging observations (Aschwanden et al. 1999; Nakariakov et al. 1999; Schrijver et al. 2002; Yuan \& Van Doorsselaere 2016a), or as Doppler shift oscillations in the spectroscopic measurements (Tian et al. 2012; Yuan \& Van Doorsselaere 2016b; Li et al. 2017b). The oscillations with large amplitudes are normally damped very rapidly, usually within several cycles (e.g., Nakariakov et al. 1999; Zimovets \& Nakariakov 2015; Goddard \& Nakariakov 2016), while those oscillations with small amplitudes could last for tens of cycles without significant damping (Nisticò et al. 2013; Anfinogentov et al. 2015). Kink oscillations of coronal loops are perturbations to the plasma's bulk parameters (Nakariakov et al. 2016). Therefore, they are helpful to remotely diagnose the coronal plasma and infer the coronal magnetic field strength. This new technique - MHD coronal seismology (Nakariakov \& Verwichte 2005; De Moortel \& Nakariakov 2012; Anfinogentov et al. 2015; Yuan \& Van Doorsselaere 2016a,b) - could improve our understanding of the coronal heating and magnetic reconnection theory (Nakariakov et al. 1999, 2016; Van Doorsselaere et al. 2016).

Kink oscillations of coronal loops are mostly observed with imaging instruments (e.g., Nisticò et al. 2013; Anfinogentov et al. 2015; Zimovets \& Nakariakov 2015; Goddard \& Nakariakov 2016) or in the warm coronal lines of spectroscopic measurements (Tian et al. 2012). In this paper, we investigate the non-damping oscillations in the Fe xxI $1354.08 \AA$ line and attempt to apply the coronal MHD seismology technique to the coronal loops. This event is jointly observed by the Interface Region Imaging Spectrograph (IRIS, De Pontieu et al. 2014), the Atmospheric Imaging Assembly (AIA, Lemen et al. 2012), and the Helioseismic and Magnetic Imager (HMI, Schou et al. 2012) on board the Solar Dynamics Observatory (SDO), the Fermi/Gamma-ray Burst Monitor (GBM, Meegan et al. 2009), and the Geostationary Operational Environment Satellites (GOES, Aschwanden 1994).

\section{Observations and data reductions}

An M 1.1 flare was detected at active region NOAA 12157 on 2014 September 6. It lasted from 16:50 UT to 17:22 UT. This flare was observed simultaneously by several instruments (see Table 1 for instrumentation). The light curves in Fig. 1a reveal the onset (marked by a vertical solid line) and two peaks during the solar flare, which might indicate the dual episodes of energy release during this event (Tian et al. 2015; Polito et al. 2016; Li et al. 2017a). In this study, we focus on the time intervals between these two episodes, from 16:58 UT (dashed line) to 17:12 UT.

Figure 1 panels $\mathrm{b}-\mathrm{f}$ show the nearly simultaneous snapshots with the same field of view (FOV) of about $50^{\prime \prime} \times 50^{\prime \prime}$ provided by the IRIS Slit-Jaw Imager (SJI), the HMI line-of-sight (LOS) magnetogram and AIA EUV/UV images. The AIA and HMI images were processed with the solar software (SSW) routines "aia_prep.pro" and "hmi_prep.pro", respectively (Lemen et al.
Table 1. Metrics of the instrumentation.

\begin{tabular}{ccccc}
\hline \hline Instruments & Channels & Cadence (s) & Pixel size & Bands \\
\hline IRIS/SP & Fe xxi $1354.08 \AA$ & 9.5 & $\sim 0.166^{\prime \prime}$ & FUV \\
IRIS/SJI & $1400 \AA$ & 19 & $\sim 0.166^{\prime \prime}$ & FUV \\
SDO/AIA & $94 \AA$ & 12 & & EUV \\
& $131 \AA$ & 24 & $\sim 0.6^{\prime \prime}$ & EUV \\
& $1600 \AA$ & 24 & & UV \\
Fermi/GBM & $4-11 \mathrm{keV}$ & & & SXR \\
& $11-26 \mathrm{keV}$ & 0.256 & - & SXR/HXR \\
& $26-50 \mathrm{keV}$ & & & HXR \\
GOES & $1-8 \AA$ & $\sim 2.0$ & - & SXR \\
SDO/HMI & $6173 \AA$ & 45 & $\sim 0.6^{\prime \prime}$ & LOS \\
\hline
\end{tabular}
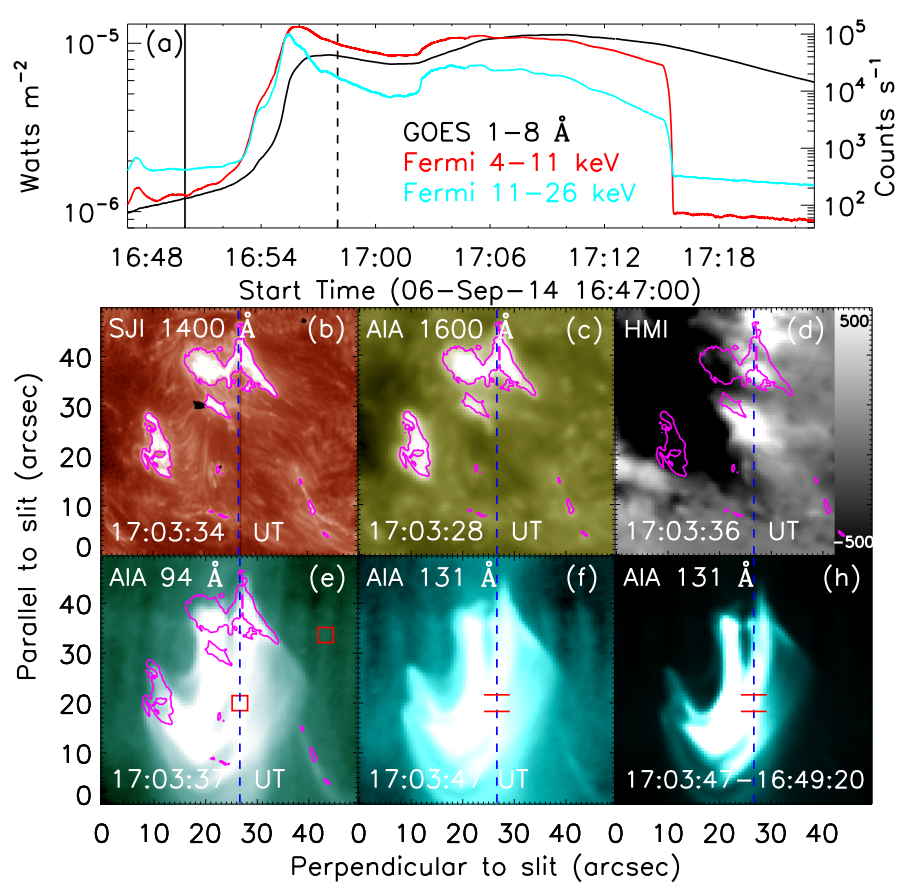

Fig. 1. Overview of the solar flare on 2014 September 6. Panel a: light curves in GOES 1-8 A (black), and Fermi 4-11 keV (red) and $11-26 \mathrm{keV}$ (turquoise). The vertical line indicates the flare onset time, the dashed line outlines the beginning time of the non-damping oscillations. Panels $b-f$ : SJI $1400 \AA$ image, AIA $1600 \AA$ image, HMI LOS magnetogram, and AIA $94 \AA$ and $131 \AA$ images. The purple contours are based on the intensity level of the SJI $1400 \AA$ image, the HMI image saturates at $\pm 500 \mathrm{G}$, and the AIA and SJI images are shown in a logarithmic brightness scale. Panel $h$ : difference image in AIA $131 \AA$. The IRIS slit position is marked by blue dashed lines. The red boxes give the regions used in the DEM analysis. Two red bars enclose the flaring loop top used for further analysis. The temporal evolution over $\sim 34$ min in AIA $94 \AA$ and $131 \AA$ is available as a movie, which also contains running difference images in the lower panels.

2012; Schou et al. 2012). The AIA $1600 \AA$ image was used as a reference to co-align with the SJI $1400 \AA$ image (Li et al. 2014, 2016b; Cheng et al. 2015), because they both contain the main bright features dominated by the continuum emission from the temperature minimum (see the contours). The bottom panels in Fig. 1 illustrate that the double flare ribbons rooted at positive and negative polarities (panel d) are connected by a bundle that has a loop structure (panel f). This agrees well with the standard solar flare model (Carmichael 1964; Sturrock 1966; Hirayama 1974; Kopp \& Pneuman 1976). Figure 1h highlights the loop 

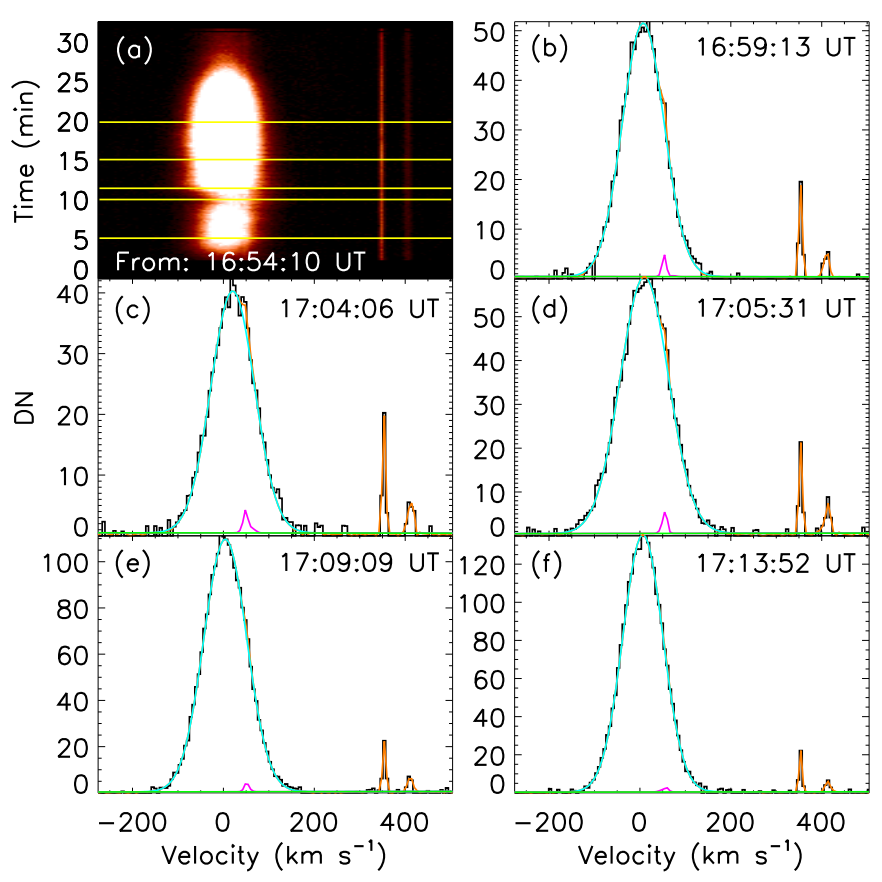

Fig. 2. Flaring spectra observed by the IRIS. Panel $a$ : time evolution of the line profiles in Fe xxı $1354.08 \AA$. Panels $b-f$ : spectral line profiles (black) and their double Gaussian fitting results (orange) with a linear background (green) at the time indicated by the yellow lines in panel $a$. The turquoise line is Fe xxı $1354.08 \AA$, and the purple line is $C_{\text {I }} 1354.29 \AA$. The zero velocity is set to the rest wavelength of Fe xxi $1354.08 \AA$.

structure with the difference image taken before and during the flare time in the AIA $131 \AA$ band. A bundle of flaring loop structures are visualized, which is very likely to be associated with hot plasma structures at about $11 \mathrm{MK}$ (Lemen et al. 2012). The flaring loop is estimated to have a length of about $48 \mathrm{Mm}$ and a width of about $5.9 \mathrm{Mm}$; the loop top reaches a height of about 25-35 Mm.

The IRIS spectra measure the flare in a "sit-and-stare" mode with a roll angle of $45^{\circ}$. The spectral scale is $\sim 25.6 \mathrm{~m} \AA$ per pixel in the far-ultraviolet (FUV) wavelengths. The IRIS slit crosses the flaring loop and one ribbon (Fig. 1). Two red bars enclose the flaring loop region used to study the quasi-periodic oscillations in this work. IRIS spectrum was pre-processed with the SSW routines of "iris_orbitval_corr_12.pro" (Tian et al. 2014; Cheng et al. 2015) and "iris_prep_despike.pro" (De Pontieu et al. 2014). To improve the signal-to-noise ratio, we apply a running average over five pixels to the IRIS spectra along the slit (Tian et al. 2012, 2016). We also manually perform the absolute wavelength calibration using a relatively strong neutral line, O I $1355.60 \AA$ (see De Pontieu et al. 2014; Tian et al. 2015; Tian 2017). IRIS observations show that Fe XXI 1354.08 $\AA$ is a hot $(\sim 11 \mathrm{MK})$ and broad emission line and is always blended with many narrow chromospheric lines at the flaring ribbons $(\mathrm{Li}$ et al. 2015b, 2016b; Tian et al. 2015; Young et al. 2015; Brosius et al. 2016; Polito et al. 2016). However, the Fe XxI $1354.08 \AA$ line is much stronger than those blended emission lines at the flaring loops (Tian et al. 2016). Figure 2 a gives the time evolution of the line profiles of Fe xxi $1354.08 \AA$, averaged over the slit positions between $\sim 18.3^{\prime \prime}$ and 21.6". Figure 2 panels $b-f$ show the spectral line profiles at the time indicated by the yellow lines in panel a. We can see that only the cool line of
C I $1354.29 \AA$ is blended with the hot line of Fe XxI $1354.08 \AA$, but its contribution is negligible. Therefore, double Gaussian functions superimposed on a linear background are used to fit the IRIS spectra at "O I" window (Tian et al. 2016). Next, we can extract the hot line of Fe XxI $1354.08 \AA$, as shown by the turquoise profile. The purple profile is the cool line of $\mathrm{C}_{\mathrm{I}} 1354.29 \AA$. Two orange peaks represent the cool lines of O I $1354.60 \AA$ and C I 1354.84 $\AA$ (Tian 2017), which are far away from the flaring line of Fe xxı 1354.08 $\AA$. Finally, the line properties of Fe xxI $1354.08 \AA$ are extracted from the fitting results, that is, Doppler velocity, peak intensity, and line width (Li et al. 2016b; Tian et al. 2016; Tian \& Chen 2018).

Figure 3 shows the time-distance (TD) images of Fe XXI $1354.08 \AA$ from the IRIS spectral fitting results, including peak intensity (a), Doppler velocity (c), and line width (e). In panel c, we can clearly see that the Doppler velocities are redshifted in the IRIS slit positions between around $10^{\prime \prime}-30^{\prime \prime}$, and these regions are located at the flaring loops, while the strong blueshifted regions correspond to the flaring ribbons (see Fig. 1). The Doppler shift oscillations exhibit two periodic behaviors. One is characterized by a series of vertical slashes with a short period near one minute, another one shows a repeating blobby pattern with a lone period of roughly five minutes. The Doppler shift oscillations with a short period appear at the redshifted wings and tend to drift downward along the IRIS slit, indicating the expansion of flaring loops. The oscillations appear to be largely coherent over a wide range along the slit of IRIS, suggesting the oscillations of a fat flaring loop, or implying that many thin flaring loops oscillate as a whole (Tian et al. 2016). Similar visible oscillations with short periods are not detected in the peak intensity and line width. We then plot the detrended images by removing a three-minute running average (Wang et al. 2009; Tian et al. 2012) of peak intensity (b) and line width (f) in Fe xxI $1354.08 \AA$; we also give the detrended image in AIA $131 \AA$ (d), which is from the IRIS slit positions. The dark vertical bars at 10, 16, 20, 24 (and so on) minutes in panel $\mathrm{d}$ are caused by the long exposure time of AIA. This is because AIA will change its exposure time when a solar flare erupts in the EUV bandpasses (Lemen et al. 2012). The detrended images in the peak intensity of Fe xxI and AIA $131 \AA$ show obvious propagating features. The speed is estimated to be about $45 \mathrm{~km} \mathrm{~s}^{-1}$, as indicated by the green arrows. This is much smaller than the local sound speed, about $500 \mathrm{~km} \mathrm{~s}^{-1}$ at $11 \mathrm{MK}$ (Nakariakov \& Ofman 2001; Kumar et al. 2013; Li et al. 2017b). It could be associated with intermittently evaporated plasma as can be seen from the movie.mp4, which clearly shows the flaring loops in AIA $94 \AA$ and $131 \AA$ propagating and intermittently passing the slit of IRIS. The detrended image of the line width does not show the apparent propagating features.

\section{Results}

\subsection{Time series}

Figure 4 panels a and b plot the time series of Doppler velocity (black), line width (green), and peak intensity (orange) in FexxI $1354.08 \AA$, as well as the normalized intensity in AIA $131 \AA$ (purple). They are averaged over 20 pixels between $18.3^{\prime \prime}$ and 21.6" (two red bars in Figs. 1f, $h$ and $3 \mathrm{c}$ ) along the IRIS slit, which cover the pronounced oscillatory behaviors. The primary behavior for the Doppler velocity seems to be the oscillations with a period of around three minutes, but such oscillations are not visible in the line width, as shown in panel a. 

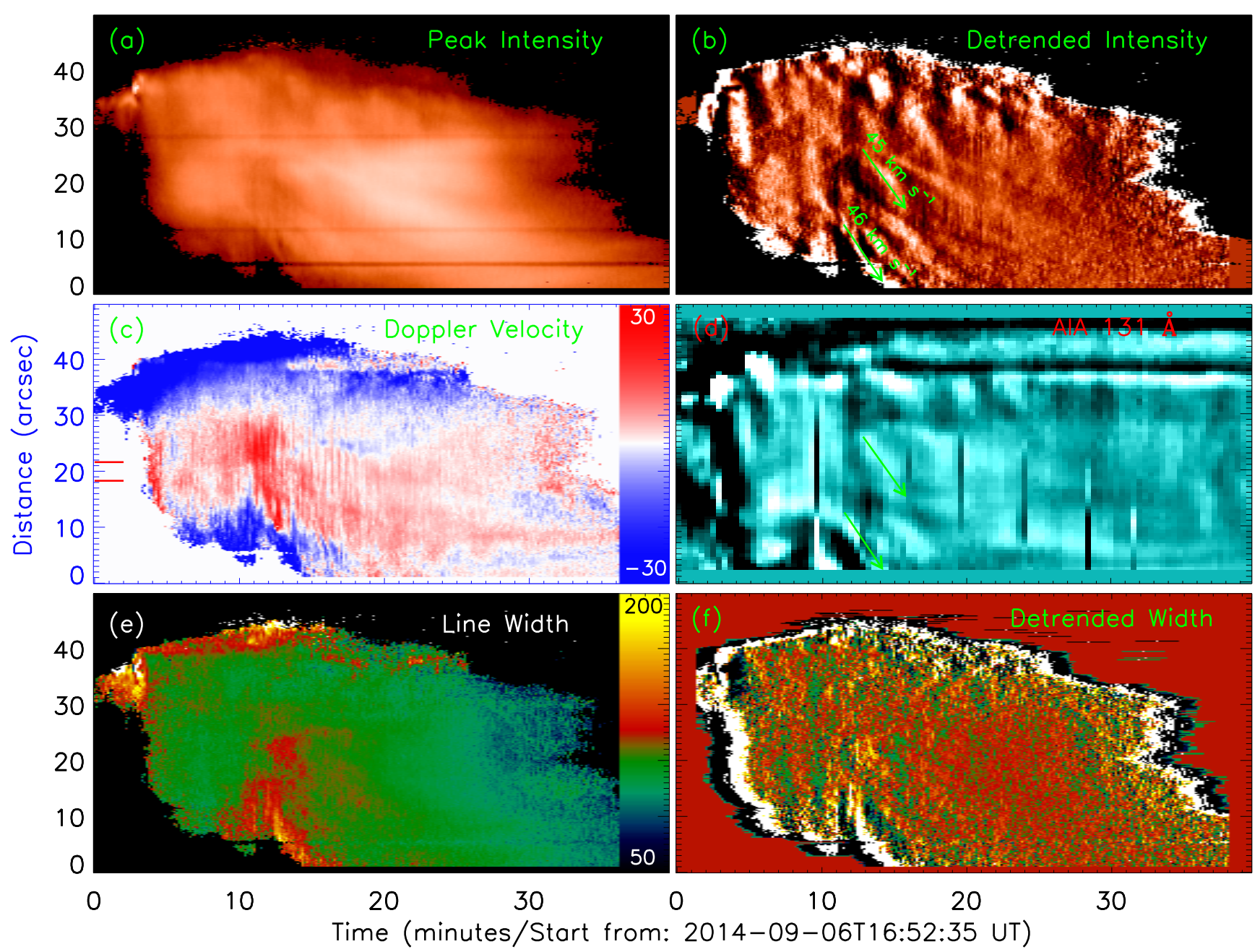

Fig. 3. Time-distance plot. Left: the peak intensity image (panel a) is shown in logarithmic scale, while the unit of color bars is shown in Doppler velocity (panel c) and line width (panel e) images in $\mathrm{km} \mathrm{s}^{-1}$. Right: detrended images in peak intensity (panel b), AIA $131 \AA ̊ 8$ intensity (panel $d$ ), and line width (panel $f$ ). Two red bars enclose the flaring loop-top region, and the green arrows indicate the propagating directions.

Moreover, the primary oscillations with 3 min period are not pronounced in the Doppler shift image in Fig. 3c. On the other hand, there are wiggles on both Doppler velocity and line width that are superimposed on their strong backgrounds, respectively. These wiggles might be the small-amplitude oscillations with a period of around one minute, which correspond well with the redshifted vertical slashes in Fig. 3c. The peak intensity and the AIA $131 \AA$ intensity exhibit two flat peaks and appear to match well, as shown in panel b. We also plot the HXR light curves in GOES 1-8 $\AA$ derivative (turquoise) and Fermi/GBM 26-50 keV (blue). Similar to the SXR fluxes in Fig. 1a, the HXR emissions also exhibit double pulses, but the second pulse is much weaker that the first one, as indicated by the blue arrow. These HXR light curves do not show the pronounced oscillatory behaviors with a periods of about three minutes. As in the Doppler velocity and line width of Fe XXI 1354.08 $\AA$, there are a series of wiggles on these HXR fluxes, which are superimposed on the strong background emissions too.

As described above, the wiggles that might be the smallamplitude oscillations are much weaker than their background emissions, which make them difficult to be identified (Dolla et al. 2012; Li \& Zhang 2017). To make these rapid oscillations more apparent, the detrended Doppler velocity and line width are accomplished by removing the $60 \mathrm{~s}$ running average from their time series (Wang et al. 2009; Tian et al. 2012; Li et al. 2017b), since we thereby enhance the short period oscillation and suppress the long period trend (Gruber et al. 2011; Auchère et al. 2016). The $60 \mathrm{~s}$ average is selected because the Doppler shift image in Fig. 3c exhibits a series of vertical slashes with a period near one minute. Fig. 5a shows that both the detrended Doppler velocity and line width exhibit the rapid oscillations with small-scale amplitudes. We also give the detrended time series of Fe XXI $1354.08 \AA$ peak intensity (orange) and AIA 131 intensity (purple), as well as the detrended HXR fluxes in GOES 1-8 (turquoise) derivative and Fermi/GBM 26-50 keV (blue), as shown in Fig. 5b. The detrended HXR fluxes and peak intensity of Fe xxı 1354.08 ^ also display the rapid oscillations; their oscillatory amplitudes are small. However, the detrended intensity in AIA $131 \AA$ does not exhibit the similar rapid oscillations, which is caused by the lower time cadence of AIA $131 \AA$ (i.e., 24 s).

\subsection{Fourier spectra and wavelet analysis}

To examine the period of flaring loop oscillations, we obtain the Fourier spectra (Fig. 6 panels a-f) with the LombScargle periodogram (Scargle 1982; Yuan et al. 2011) for the 


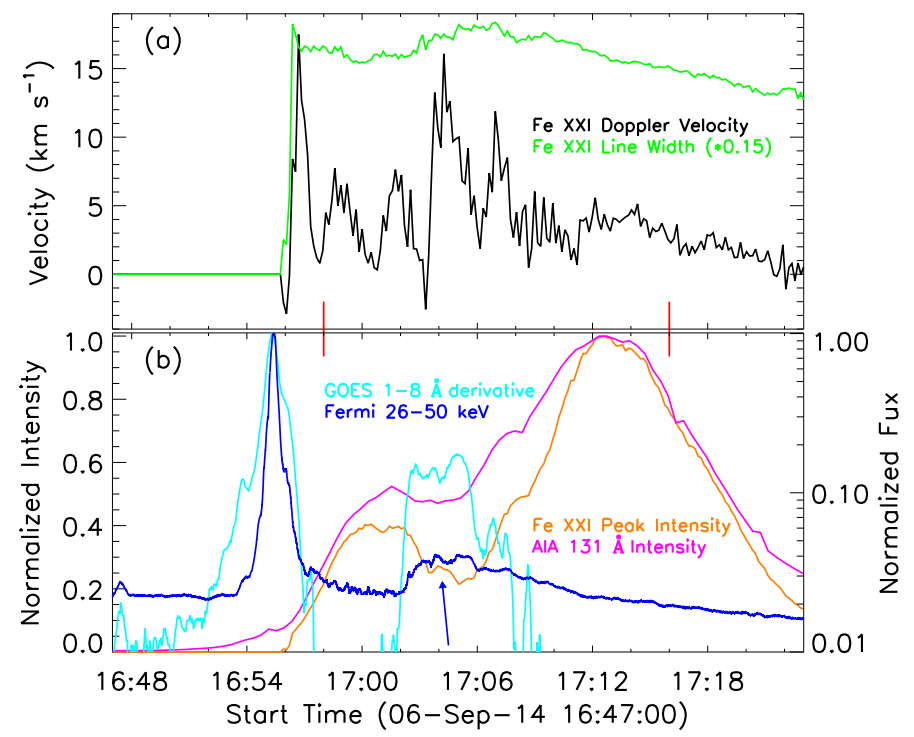

Fig. 4. Time-evolution curves. Time series of Doppler velocity (black), line width (green), and peak intensity (orange) in Fe xxi $1354.08 \AA$, as well as the AIA $131 \AA$ intensity (purple), the light curves in GOES 1-8 A derivative (turquoise), and Fermi $26-50 \mathrm{keV}$ (green). The blue arrow indicates a small HXR pulse, and two red vertical ticks outline the detrended time series in Fig. 5.

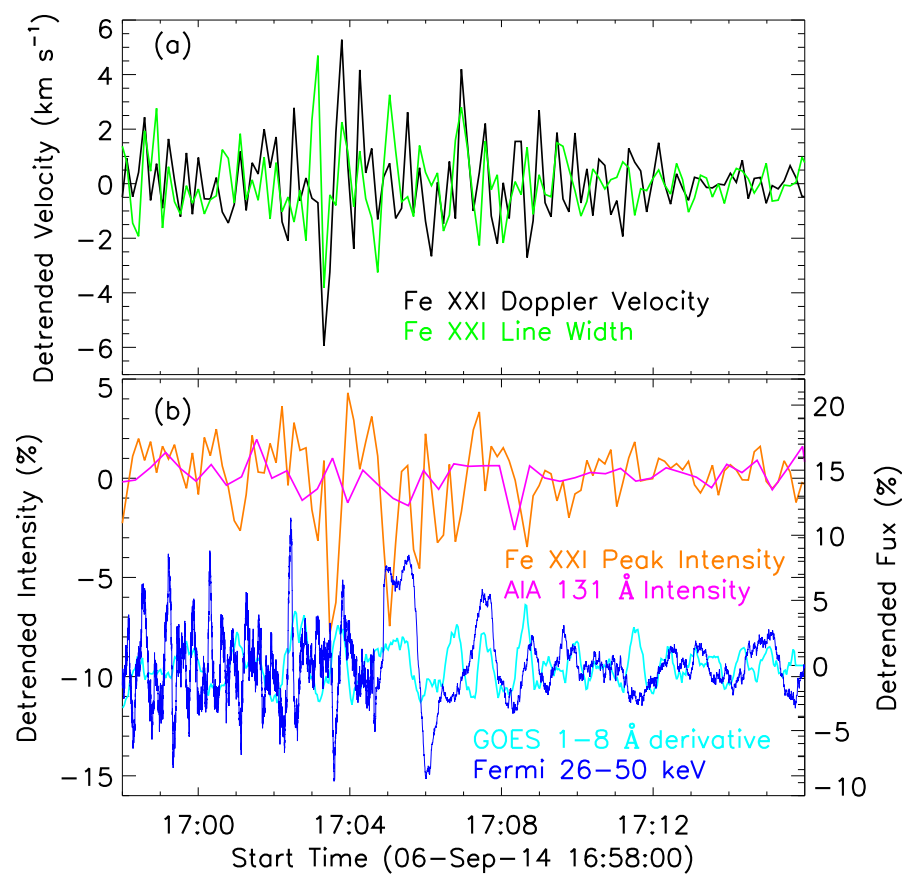

Fig. 5. Detrended time-evolution profiles. Detrended time series of the curves in Fig. 4 between 16:58 UT and 17:16 UT.

detrended time series of Doppler velocity, line width, peak intensity in FexxI $1354.08 \AA$, and AIA $131 \AA$ intensity, as well as the detrended HXR fluxes in GOES $1-8 \AA$ derivative and Fermi $26-50 \mathrm{keV}$. Then the dominant period is determined from the peak value of the power spectrum, while the error bar is determined as the full-width-at-half-maximum value of the peak power (Yuan et al. 2011; Tian et al. 2016; Li et al. 2017b). Figure 6 panel a shows that there are two distinct peaks above

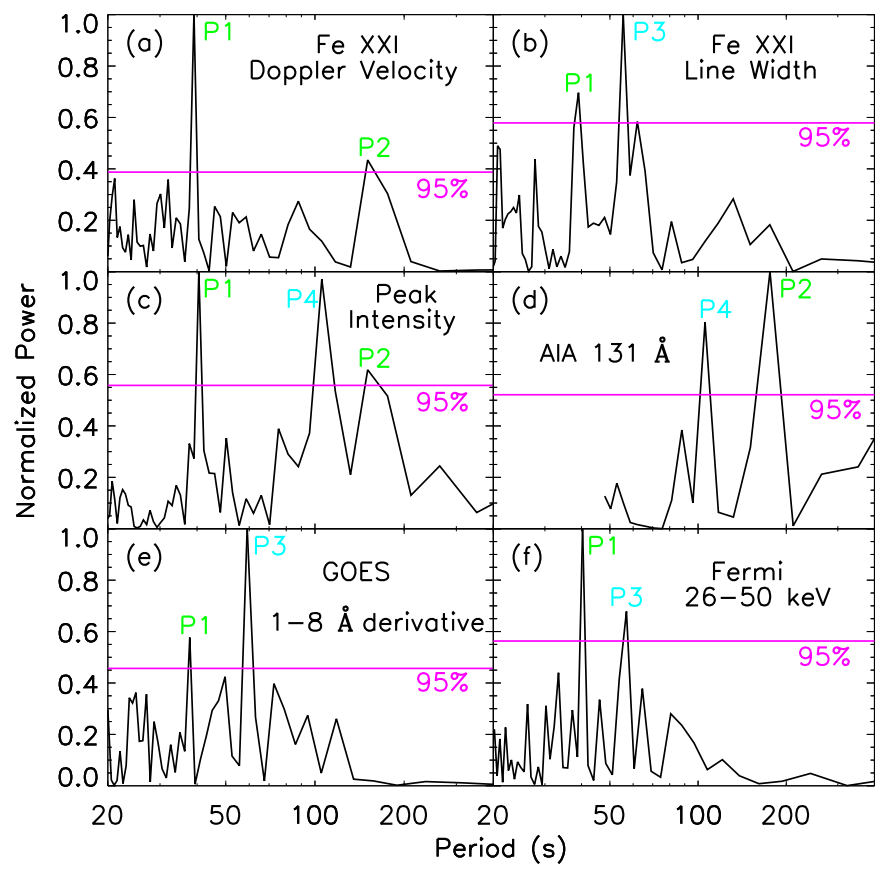

Fig. 6. Fourier spectra. Normalized FFT power spectra of the detrended time series from Doppler velocity (panel $a$ ), line width (panel $b$ ), and peak intensity (panel c) in Fe xxi 1354.08 ̊., AIA EUV (panel d), GOES derivative (panel e), and Fermi HXR (panel f) fluxes. A horizontal purple line in each panel indicates the $95 \%$ confidence level.

the $95 \%$ confidence level (Horne \& Baliunas 1986). Therefore, two prominent periods were obtained in the Doppler velocity of Fexxi $1354.08 \AA, 40 \pm 3 \mathrm{~s}$ (P1) and $155 \pm 15 \mathrm{~s}$ (P2). The shorter period (P1) is also detected in the line width and peak intensity of Fe Xxı $1354.08 \AA$, and the HXR emissions in GOES 1-8 $\AA$ derivative and Fermi $26-50 \mathrm{keV}$. However, the AIA $131 \AA$ intensity does not exhibit the shorter period (P1), because of its lower cadence (24s). On the other hand, the longer period (P2) is observed in the Doppler velocity, peak intensity of Fe xxi $1354.08 \AA$, and AIA $131 \AA$ intensity. Besides the two dominant periods, we also detect another two periods from the power spectrum. For example, a period $(\mathrm{P} 3=55 \pm 5 \mathrm{~s})$ is identified in the line width of Fe XxI 1354.08 $\AA$, as well as in the HXR emissions in GOES $1-8 \AA$ derivative and Fermi $26-50 \mathrm{keV}$. Another period $(\mathrm{P} 4=110 \pm 10 \mathrm{~s})$ is found in the peak intensity of Fe xxI $1354.08 \AA$ and AIA $131 \AA$ intensity.

We also perform wavelet analysis (Torrence \& Compo 1998; Yuan et al. 2011; Deng et al. 2012; Tian et al. 2016) on the detrended time series of Doppler velocity, line width, and peak intensity in Fe xxI 1354.08 $\AA$ and the detrended HXR fluxes in Fermi 26-50 keV. Figure 7 gives their wavelet power spectra (left) and global wavelet (right), respectively. They show similar periods to the Fourier analysis results, and the major oscillations occurs from 17:00 UT to 17:12 UT; this is the time after the first SXR peak or HXR pulse. However, the dominant periods in the wavelet power spectra and global wavelet spectra are always with a broad band, which makes some close periods mix together, such as P1 and P3 in panels b and d, and P2 and P4 in panel c. The oscillatory amplitudes, determined with the square root of the peak global wavelet power, are $2.2 \mathrm{~km} \mathrm{~s}^{-1}$ for the Doppler velocity, $1.9 \mathrm{~km} \mathrm{~s}^{-1}$ for the line width, and $3.6 \%$ for the peak intensity. 


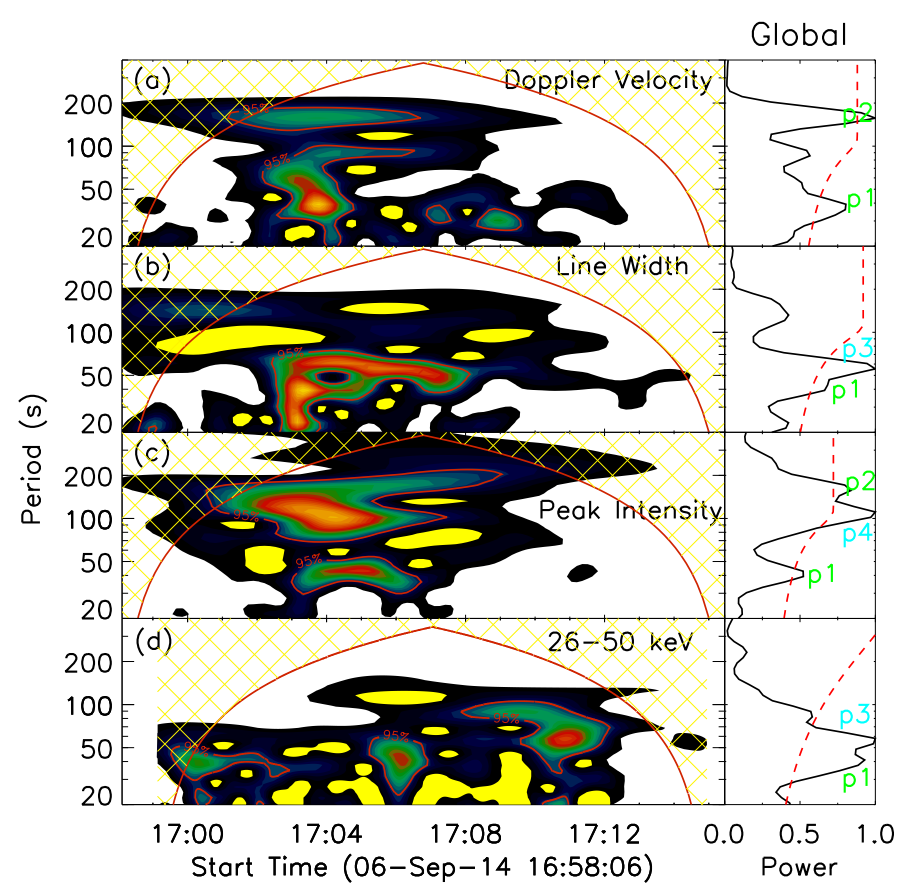

Fig. 7. Wavelet analysis results. Wavelet power spectra and global wavelet of the detrended time series from Fe Xxı 1354.08 A Doppler velocity (panel $a$ ), line width (panel $b$ ), and peak intensity (panel $c$ ), as well as Fermi $26-50 \mathrm{keV}$ (panel d). The red lines indicate a significance level of $95 \%$.

\subsection{Cross-correlation and DEM analysis}

The cross-correlation analysis (Deng et al. 2013; Tian et al. 2016) is applied to investigate the time lag between Doppler velocity and peak intensity in Fe Xxi $1354.08 \AA$, as shown by the red line in Fig. 8a. A maximum correlation coefficient is found at the time lag of about $10 \mathrm{~s}$. The maximum correlation is associated with the $40 \mathrm{~s}$ oscillatory signal, so the time lag corresponds to a phase shift of about $\pi / 2$. Our finding in a hot flaring line is similar to those in the warm coronal emission lines (Tian et al. 2012). Moreover, the same phase shift $(\pi / 2)$ can also be found between the Doppler velocity signal in the Fe XxI $1354.08 \AA$ line and the detrended HXR fluxes in Fermi $26-50 \mathrm{keV}$ (turquoise line). On the other hand, the maximum correlation coefficient is found at the time lag of around $0 \mathrm{~s}$ between the detrended time series of Doppler velocity and line width in Fe XxI $1354.08 \AA$ (black line), indicating that the line width oscillates in phase with the Doppler velocity. The same phase oscillations can also be detected between the peak intensity in Fe xxI 1354.08 $\AA$ and HXR fluxes in $26-50 \mathrm{keV}$, as seen in the green line.

Using AIA intensity images in six EUV bandpasses, a differential emission measure (DEM) analysis (Cheng et al. 2012; Shen et al. 2015) is performed for the solar flare at 17:03:37 UT, when the oscillations are pronounced. Figure 8 panels $b$ and $c$ plot the DEM profiles in the flaring loop and the background corona, respectively. They contain the same region with an FOV of $3^{\prime \prime} \times 3^{\prime \prime}$, as enclosed by the red boxes in Fig. 1e. The black profile in each panel is the best-fit DEM solution to the observed fluxes. The colored rectangles represent the errors of the DEM curve, which are calculated from 100 Monte Carlo (MC) realizations of the observational data (Cheng et al. 2012; Tian et al. 2016; $\mathrm{Li}$ et al. 2017b). The average temperature $(T)$ and emission measure (EM) inside and outside (background corona) of the flaring loop are also estimated according to their errors,
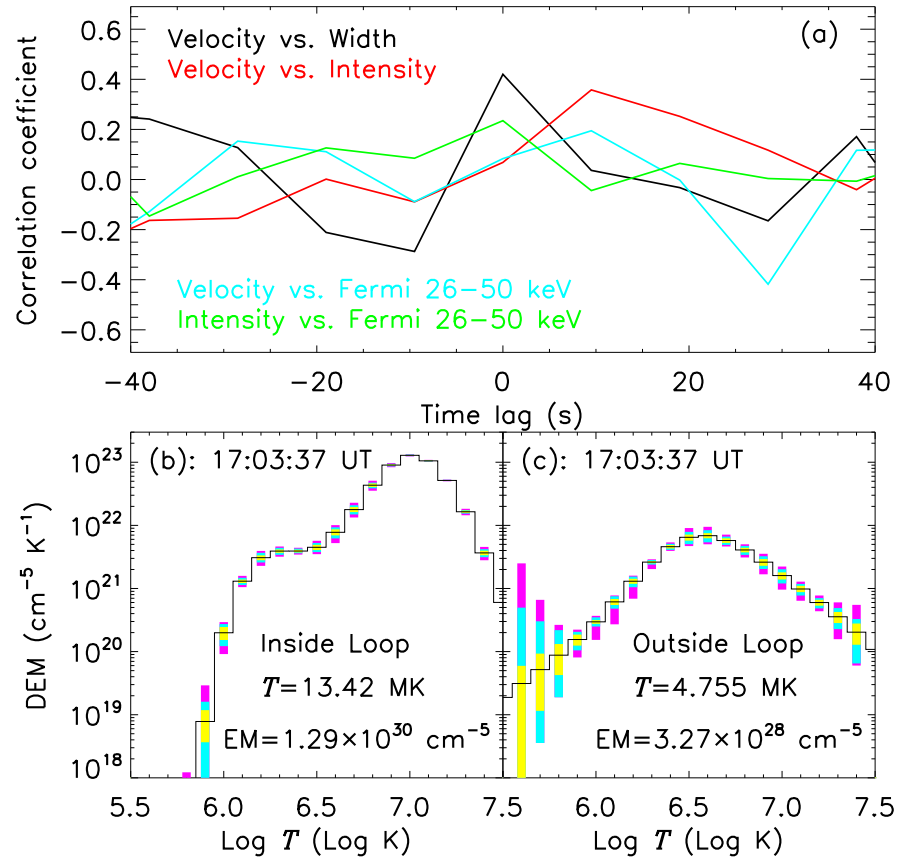

Fig. 8. Cross-correlation and DEM analysis results. Panel a: correlation coefficient $(\mathrm{cc})$ between two parameters as a function of time lag. (panels $b$ and $c$ ): DEM profiles in the flaring loop (panel $b$ ) and background corona (panel c) marked by the red boxes in Fig. 1. The black profile is the best-fitted DEM curve from AIA observations. The rectangles with different colors on the DEM plot encompass 95\% (purple), $80 \%$ (turquoise), and 50\% (yellow) of the Monte Carlo solutions, respectively. The mean temperature, EM, and observed time are labeled in each panel.

respectively. For example, the confident temperature $(\log T)$ range inside the flaring loop is $6.0-7.5$, while that outside of the flaring loop is 5.8-7.1, since the temperatures in solar flare are much higher than that in the background corona. Therefore, the number density inside the flaring loop can be estimated with $n_{e}=\sqrt{E M / w}$ by assuming a filling factor of 1.0 (Tian et al. 2016; Li et al. 2017b), and we can obtain a lower limited density inside the flaring loop of $\sim 4.7 \times 10^{10} \mathrm{~cm}^{-3}$. On the other hand, the effective LOS depth $\left(l \approx \sqrt{H \pi r} \sim 4 \times 10^{10} \mathrm{~cm}\right)$, instead of the loop width, is applied to calculate the number density outside of the flaring loop (Zhang \& Ji 2014; Zucca et al. 2014; Su et al. 2016; $\mathrm{Li}$ et al. 2017b), and we get $9.1 \times 10^{8} \mathrm{~cm}^{-3}$. Finally, a number density ratio $\left(r_{\mathrm{d}}=n_{0} / n_{\mathrm{e}}\right)$ of $\sim 0.02$ between outside and inside of the flaring loop is determined, which is very close to the density contrast from recent observations (Tian et al. 2016; Li et al. 2017b).

\subsection{Magnetic field modeling}

To determine the magnetic field strength of the flaring loops, we construct magnetic field models using the flux rope insertion method developed by van Ballegooijen (2004). We briefly introduce the method below, and detailed descriptions can be found in Bobra et al. (2008) and Su et al. (2009, 2011). First, a potential field model is computed from the high-resolution (HIRES) and global magnetic maps observed by SDO/HMI. The lower boundary condition for the HIRES region is derived from the photospheric line-of-sight magnetograms obtained at 16:36 UT on 2014 September 6 . The longitude-latitude map of the radial component of the magnetic field in the HIRES region is presented in 

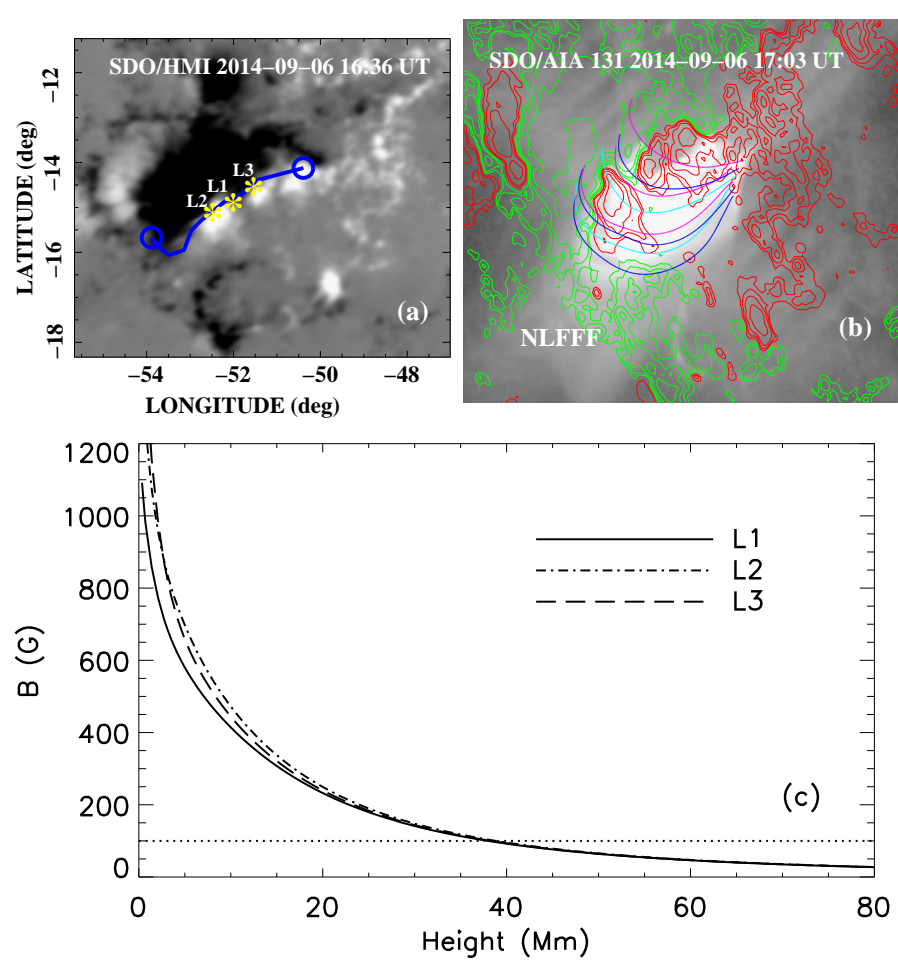

Fig. 9. Magnetic field strength estimated from the NLFFF model Panel a: zoomed view of the longitude-latitude map of the radial component of the photospheric magnetic field by SDO/HMI in the HIRES region at 16:36 UT on 2014 September 6. The blue curve with circles at the two ends refers to the path along which we insert the flux rope. Panel $b$ : selected field lines from the NLFFF model overlaid on a flare image by AIA, and the red (green) contours refer to the positive (negative) photospheric magnetic fields by HMI. Panel $c$ : the distributions of the magnetic field strength from this model with height at three locations, L1, L2, and L3 (marked with yellow stars on panel a.

Fig. 9 panel a. The HIRES computational domain extends about $29^{\circ}$ in longitude, $28^{\circ}$ in latitude, and up to $1.7 R_{\odot}$ from the Sun center. The models use variable grid spacing to achieve high spatial resolution in the lower corona (i.e., $0.001 R_{\odot}$ ) while covering a large coronal volume in and around the target region. Next we modify the potential field to create cavities in the region above the selected path marked with a blue curve, then insert one thin flux bundle (representing the axial flux $\Phi_{\text {axi }}$ of the flux rope) into the cavities. Circular loops are added around the flux bundle to represent the poloidal flux $F_{\text {pol }}$ of the flux rope. The resulting magnetic fields are not in force-free equilibrium. We then use the magneto-frictional relaxation to drive the field towards a force-free state (van Ballegooijen et al. 2000; Yang et al. 1986).

We construct a series of magnetic field models by varying the axial and poloidal fluxes of the inserted flux ropes. One of the best-fit non-linear force-free field (NLFFF) models is presented in Fig. 9 panel b. The inserted flux bundle has a poloidal flux of $0 \mathrm{Mx} \mathrm{cm}^{-1}$, and the axial flux is $4 \times 10^{20} \mathrm{Mx}$. We can see that selected model field lines match the observed post flaring loops well. The distributions of the magnetic field strength from this model with height at three locations, L1, L2, and L3, are presented in Fig. 9 panel c. We can see that the difference of the magnetic strength at these three locations decreases with height. They all reach more than $100 \mathrm{G}$ at a height of $\sim 35 \mathrm{Mm}$ above the photosphere. Similar results are also obtained from the potential field model.

\section{Discussions}

Four distinct periods are identified from the FFT power spectra, as shown in Fig. 6. The short periods (P1 and P3) can be detected simultaneously by the IRIS (a, b, c), GOES (e), and Fermi/GBM (f), which could exclude the instrument affects. Moreover, they can be clearly seen as a series of vertical slashes near one minute in the redshifted wing of the Doppler velocity image (Fig. 3c). On the other hand, such small-scale oscillations have been reported using the Hinode/EIS or IRIS observations (see Kitagawa et al. 2010; Tian et al. 2012, 2016), which indicated that we could detect the small-amplitude oscillations, especially in the Doppler velocity images. The longer periods ( $\mathrm{P} 2$ and $\mathrm{P} 4)$ can also be observed simultaneously by IRIS and SDO/AIA. They are the primary behaviors for the time series of Doppler velocity (black line) in Fig. 4a, which seem to oscillate with a period of about two-three minutes. Therefore, these periods obtained by the FFT method are reliable.

Usually, quasi-periodic oscillations observed in the impulsive phase of a solar flare are thought to be modulated by the injection of non-thermal electrons accelerated by quasi-periodic magnetic reconnection (e.g., Dolla et al. 2012; Li et al. 2015a). However, the quasi-periodic oscillations in this study are more likely to be associated with MHD waves, as they are detected after the first HXR pulse, which endures the passage from impulsive to decay phases of a solar flare. The phase speed of the non-damping oscillations $\left(c_{\mathrm{p}}=2 L / P\right)$ could be estimated to be $\sim 1200 \mathrm{~km} \mathrm{~s}^{-1}$, which is larger than the local sound speed $\left(\sim 500 \mathrm{~km} \mathrm{~s}^{-1}\right)$ at $11 \mathrm{MK}$. Therefore, they are not standing slow waves. Sausage waves can be excluded, because they are often thought to have no Doppler shift oscillations (Kitagawa et al. 2010), or that the line width oscillation period is half of the intensity or velocity oscillation period (Jess et al. 2008). On the other hand, kink waves can be detected in the intensity disturbances when the LOS is not perpendicular to loop displacement (Tian et al. 2012; Wang et al. 2012; Zimovets \& Nakariakov 2015; Yuan \& Van Doorsselaere 2016b). Figure 1 shows that the slit of IRIS is not exactly perpendicular with the flaring loop, thus we can observe the quasi-periodic oscillations (P1) from the Doppler velocity, the line width, and the peak intensity in Fe XXI 1354.08 $\AA$. The quasi-periodic oscillations in the line width of Fe XXI $1354.08 \AA$ indicate the temporal variations in temperature broadening of the iron line. The analysis from crosscorrelation reveals a $\pi / 2$ phase shift between Doppler velocity and peak intensity signals, which may be caused by the periodic crossing of the flaring loops over the IRIS slit (Tian et al. 2012, 2016). The quasi-periodic oscillations can also be clearly seen from the HXR emissions in GOES $1-8$ Å derivative and Fermi $26-50 \mathrm{keV}$. These HXR fluxes integrated over the entire Sun are also found to be in-phase oscillations with the spatially resolved peak intensity in Fe Xxi $1354.08 \AA$ (Fig. 8a). The kink wave on a coronal loop in the HXR emission was often observed as the back and forth movement of a X-ray source. However, it can be connected with the electron acceleration, which is modulated by kink oscillations of a flaring loop, or with the interaction of a flaring loop with another loop that performs kink oscillations. Thus, the reconnection field is periodically fed to the reconnection site by the kink oscillations (Nakariakov \& Verwichte 2005). All these clues suggest that the quasi-periodic oscillations with a short period (P1) at flaring loops are most likely to be the global kink mode (Uchida 1970; Asai et al. 2001; Tian et al. 2016).

The global kink oscillations with a period of $\sim 40 \mathrm{~s}$ are observed simultaneously with the line properties of a flaring line in Fe XXI $1354.08 \AA$, and their amplitudes are not damped 
significantly. This is similar to the kink oscillations detected in Doppler velocity at redshifted wings in the warm coronal lines (Tian et al. 2012). Both observations find that the kink oscillations in the peak intensity and line width exhibit a very small oscillatory amplitude. This also explains that the time-distance images of peak intensity and line width do not display the same obvious oscillations as that of Doppler velocity, because those oscillations overlap on the strong background (see also, Dolla et al. 2012; Li et al. 2017b). Our new observational result is that the kink oscillations within non-damping amplitudes are identified at hot flaring loops, at $11 \mathrm{MK}$. To our knowledge, non-damping kink oscillations in a hot flaring line (e.g., Fe XXI 1354.08 $\AA$ ) have never been reported. Previous reports of kink oscillations were mainly observed in the warm $(<10 \mathrm{MK})$ coronal lines (Tian et al. 2012; Wang et al. 2012) or EUV images (Su et al. 2012; Goddard \& Nakariakov 2016; Yuan \& Van Doorsselaere 2016a). Thanks to the high-resolution observations from the IRIS, we can investigate non-damping kink oscillations in a hot line of Fe Xxi $1354.08 \AA$ at flaring loops.

Previous observations showed that the periods of kink oscillations were usually larger than $60 \mathrm{~s}$, and even tens of minuets. However, these longer periods of kink oscillations were observed from the imaging observations in EUV/SXR passbands (Nakariakov et al. 1999; Aschwanden et al. 2002; Yuan \& Van Doorsselaere 2016a) or in spectroscopic observations in warm coronal lines (Tian et al. 2012). Actually, kink oscillations with a short period of $6.6 \mathrm{~s}$ have been reported by Asai et al. (2001) using the Nobeyama Radioheliograph, and a $43 \mathrm{~s}$ periodicity is found by Koutchmy et al. (1983) in the Doppler velocity of the Fe xIV $5303 \AA$ line, which is interpreted as a standing kink wave (Roberts et al. 1984). All those periods of kink oscillations are less than $60 \mathrm{~s}$, which are similar to our results. On the other hand, the oscillatory amplitude of peak intensity in Fe xxi $1354.08 \AA$ is smaller than $3.6 \%$, and the oscillations are not damped. This is consistent with the non-damping kink oscillations of coronal loops observed in AIA/EUV images, which also have smaller amplitudes but can last for tens of cycles (e.g., Anfinogentov et al. 2013, 2015; Nisticò et al. 2013). Therefore, the short period of kink oscillations at hot flaring loops is reasonable, because hot flaring loops are much shorter than warm coronal loops (Aschwanden et al. 2002; Anfinogentov et al. 2015).

Another short period (P3) of $\sim 55 \mathrm{~s}$ could also be kink oscillations, and oscillatory period is very close to the period of $\mathrm{P} 1$. In the wavelet power spectra, these two periods even mix together and are difficult to distinguish. The small difference between these two periods might be caused by the expansion of the flaring loops with time (Verth \& Erdélyi 2008; Tian et al. 2016). Based on the model of kink oscillations, we can estimate the magnetic fields at flaring loops from Eq. (1) (Roberts et al. 1984; Nakariakov \& Ofman 2001; Nakariakov \& Verwichte 2005; Nakariakov \& Melnikov 2009; Tian et al. 2012; Yuan \& Van Doorsselaere 2016a),

$B \approx 4.6 \times 10^{-12} \frac{L}{P_{\mathrm{k}}} \sqrt{2 n_{\mathrm{e}}\left(1+r_{\mathrm{d}}\right)}$.

As mentioned above, the length of flaring loop $(L)$, the number density in flaring loops, and the density ratio $\left(r_{\mathrm{d}}\right)$ between outside and inside flaring loops have been obtained; they are $\sim 48 \mathrm{Mm}, \sim 4.7 \times 10^{10} \mathrm{~cm}^{-3}$, and $\sim 0.02$, respectively. Therefore, the magnetic fields at flaring loops can be estimated from the periods $\left(P_{k} \sim 40-55 \mathrm{~s}\right)$ of kink oscillations, which are about $120-170 \mathrm{G}$. This is consistent with the magnetic field modeling results of $110-180 \mathrm{G}$ at a height of $\sim 25-35 \mathrm{Mm}$ above the pho- tosphere (see, Fig. 9c). Our results are also similar to previous findings obtained by Qiu et al. (2009).

Longer periods of about $155 \mathrm{~s}$ and $110 \mathrm{~s}$ are clearly seen in the peak intensity of Fe xxi $1354.08 \AA$ and AIA $131 \AA$ intensity. They are propagating with a speed of $\sim 45 \mathrm{~km} \mathrm{~s}^{-1}$ (Fig. 3). The period of around $155 \mathrm{~s}$ can also be observed in the Doppler velocity of Fe Xxi $1354.08 \AA$, and the longer periods always oscillate in the redshifted wings, which might be related to the enhancement of downflows in flaring loops after "chromospheric evaporation" (Tian et al. 2015; Li et al. 2017c). However, these longer periods are missed by the HXR fluxes in GOES $1-8 \AA$ derivative and Fermi $26-50 \mathrm{keV}$. Therefore, these longer periods cannot be considered to be the MHD waves. They are most likely the recurring downflows in flaring loops, which are caused by the flaring loops periodically crossing the slit of IRIS (Tian et al. 2012, 2016). The two domain periods in the intensity of Fe XXI $1354.08 \AA$ and AIA $131 \AA$ are probably due to the flaring loop expansions.

\section{Summary}

Using the observational data from IRIS, SDO, GOES, and Fermi, we explored the non-damping oscillations with a short period of $\sim 40 \mathrm{~s}$ in a hot flaring line of Fe XxI $1354.08 \AA$, which can be detected in the Doppler velocity, the line width, the peak intensity of Fe xxi $1354.08 \AA$, and also the HXR fluxes in GOES $1-8 \AA$ derivative and Fermi $26-50 \mathrm{keV}$. A $\pi / 2$ phase shift between the detrended time series of the Doppler velocity and peak intensity (and HXR fluxes) is found, while the Doppler velocity and line width are found to be oscillating in phase. The oscillatory amplitudes of the Doppler velocity and the line width are identified to be $2.2 \mathrm{~km} \mathrm{~s}^{-1}$, and $1.9 \mathrm{~km} \mathrm{~s}^{-1}$, respectively, while that of the peak intensity is less than $3.6 \%$ related to the peak intensity background trend. A longer period of $\sim 155 \mathrm{~s}$ is observed in the Doppler velocity, the peak intensity of Fe xxi $1354.08 \AA$, and also the AIA $131 \AA$ intensity, which is mostly likely the recurring downflows at hot flaring loops rather than the MHD waves.

Acknowledgements. The authors would like to thank the anonymous referee for his or her valuable comments. We acknowledge Prof. H. Tian for his inspiring discussions. We also thank the teams of IRIS, SDO, Fermi, and GOES for their open data use policy. This work is supported by NSFC under grants 11603077 , 11573072, 11773079, 11790300, 11729301, 11790302, 11473071, 11333009, the CRP (KLSA201708), the Youth Fund of Jiangsu Nos. BK20161095, and BK20171108, as well as the National Natural Science Foundation of China (U1731241), the Strategic Priority Research Program on Space Science, CAS, Grant No. XDA15052200 and XDA15320301. D. Li is supported by the Specialized Research Fund for State Key Laboratories. Y. N., Su is also supported by one hundred talent program of CAS. The Laboratory No. 2010DP173032. Li \& Ning also acknowledge support by ISSI-BJ to the team of "Pulsations in solar flares: matching observations and models".

\section{References}

Andries, J., Arregui, I., \& Goossens, M. 2005, ApJ, 624, L57

Anfinogentov, S., Nisticò, G., \& Nakariakov, V. M. 2013, A\&A, 560, A107 Anfinogentov, S. A., Nakariakov, V. M., \& Nisticò, G. 2015, A\&A, 583, A136 Asai, A., Shimojo, M., Isobe, H., et al. 2001, ApJ, 562, L103

Aschwanden, M. J. 1994, Sol. Phys., 152, 53

Aschwanden, M. J., Fletcher, L., Schrijver, C. J., \& Alexander, D. 1999, ApJ, 520,880

Aschwanden, M. J., de Pontieu, B., Schrijver, C. J., \& Title, A. M. 2002, Sol. Phys., 206, 99

Auchère, F., Froment, C., Bocchialini, K., Buchlin, E., \& Solomon, J. 2016, ApJ, 825,110

Bobra, M. G., van Ballegooijen, A. A., \& DeLuca, E. E. 2008, ApJ, 672, 1209 
Brosius, J. W., Daw, A. N., \& Inglis, A. R. 2016, ApJ, 830, 101

Carmichael, H. 1964, NASA Spec. Publ., 50, 451

Cheng, X., Zhang, J., Saar, S. H., \& Ding, M. D. 2012, ApJ, 761, 62

Cheng, X., Ding, M. D., \& Fang, C. 2015, ApJ, 804, 82

Deng, L. H., Qu, Z. Q., Wang, K. R., \& Li, X. B. 2012, Adv. Space Res., 50, 1425

Deng, L., Qi, Z., Dun, G., \& Xu, C. 2013, PASJ, 65, 11

De Moortel, I., \& Nakariakov, V. M. 2012, Philos. Trans. R. Soc. Lond. A, 370, 3193

De Moortel, I., Ireland, J., Hood, A. W., \& Walsh, R. W. 2002, A\&A, 387, L13

De Pontieu, B., Title, A. M., Lemen, J. R., et al. 2014, Sol. Phys., 289, 2733

Dolla, L., Marqué, C., Seaton, D. B., et al. 2012, ApJ, 749, L16

Goddard, C. R., \& Nakariakov, V. M. 2016, A\&A, 590, L5

Gruszecki, M., Nakariakov, V. M., \& Van Doorsselaere, T. 2012, A\&A, 543, A12

Gruber, D., Lachowicz, P., Bissaldi, E., et al. 2011, A\&A, 533, A61

Hirayama, T. 1974, Sol. Phys., 34, 323

Horne, J. H., \& Baliunas, S. L. 1986, ApJ, 302, 757

Inglis, A. R., \& Nakariakov, V. M. 2009, A\&A, 493, 259

Jess, D. B., Rabin, D. M., Thomas, R. J., et al. 2008, ApJ, 682, 1363

Kitagawa, N., Yokoyama, T., Imada, S., \& Hara, H. 2010, ApJ, 721, 744

Kliem, B., Dammasch, I. E., Curdt, W., \& Wilhelm, K. 2002, ApJ, 568, L61

Kopp, R. A., \& Pneuman, G. W. 1976, Sol. Phys., 50, 85

Koutchmy, S., Zhugzhda, I. D., \& Locans, V. 1983, A\&A, 120, 185

Kumar, P., Innes, D. E., \& Inhester, B. 2013, ApJ, 779, L7

Kumar, P., Nakariakov, V. M., \& Cho, K.-S. 2016, ApJ, 822, 7

Lemen, J. R., Title, A. M., Akin, D. J., et al. 2012, Sol. Phys., 275, 17

Li, Y. P., \& Gan, W. Q. 2008, Sol. Phys., 247, 77

Li, T., \& Zhang, J. 2015, ApJ, 804, L8

Li, D., \& Zhang, Q. M. 2017, MNRAS, 471, L6

Li, B., Habbal, S. R., \& Chen, Y. 2013, ApJ, 767, 169

Li, L. P., Peter, H., Chen, F., \& Zhang, J. 2014, A\&A, 570, A93

Li, D., Ning, Z. J., \& Zhang, Q. M. 2015a, ApJ, 807, 72

Li, Y., Ding, M. D., Qiu, J., \& Cheng, J. X. 2015b, ApJ, 811, 7

Li, L. P., Zhang, J., Su, J. T., \& Liu, Y. 2016a, ApJ, 829, L33

Li, D., Innes, D. E., \& Ning, Z. J. 2016b, A\&A, 587, A11

Li, D., Zhang, Q. M., Huang, Y., Ning, Z. J., \& Su, Y. N. 2017a, A\&A, 597, L4

Li, D., Ning, Z. J., Huang, Y., et al. 2017b, ApJ, 849, 113

Li, D., Ning, Z. J., Huang, Y., \& Zhang, Q. M. 2017c, ApJ, 841, L9

Mariska, J. T. 2005, ApJ, 620, L67

Mandal, S., Yuan, D., Fang, X., et al. 2016, ApJ, 828, 72

Meegan, C., Lichti, G., Bhat, P. N., et al. 2009, ApJ, 702, 791

Milligan, R. O., Fleck, B., Ireland, J., Fletcher, L., \& Dennis, B. R. 2017, ApJ, 848, L8

Nakariakov, V. M., \& Ofman, L. 2001, A\&A, 372, L53

Nakariakov, V. M., \& Verwichte, E. 2005, Liv. Rev. Sol. Phys., 2, 3

Nakariakov, V. M., \& Melnikov, V. F. 2009, Space Sci. Rev., 149, 119

Nakariakov, V. M., Ofman, L., Deluca, E. E., Roberts, B., \& Davila, J. M. 1999, Science, 285, 862

Nakariakov, V. M., Foullon, C., Myagkova, I. N., \& Inglis, A. R. 2010, ApJ, 708, L47

Nakariakov, V. M., Pilipenko, V., Heilig, B., et al. 2016, Space Sci. Rev., 200, 75

Ning, Z. 2014, Sol. Phys., 289, 1239

Ning, Z. 2017, Sol. Phys., 292, 11
Ning, Z., Ding, M. D., Wu, H. A., Xu, F. Y., \& Meng, X. 2005, A\&A, 437, 691 Nisticò, G., Nakariakov, V. M., \& Verwichte, E. 2013, A\&A, 552, A57 Ofman, L., \& Wang, T. 2002, ApJ, 580, L85

Polito, V., Reep, J. W., Reeves, K. K., et al. 2016, ApJ, 816, 89

Qiu, J., Gary, D. E., \& Fleishman, G. D. 2009, Sol. Phys., 255, 107

Roberts, B., Edwin, P. M., \& Benz, A. O. 1984, ApJ, 279, 857

Ruderman, M. S., \& Erdélyi, R. 2009, Space Sci. Rev., 149, 199

Scargle, J. D. 1982, ApJ, 263, 835

Schou, J., Scherrer, P. H., Bush, R. I., et al. 2012, Sol. Phys., 275, 229

Schrijver, C. J., Aschwanden, M. J., \& Title, A. M. 2002, Sol. Phys., 206, 69

Shen, Y., \& Liu, Y. 2012, ApJ, 753, 53

Shen, Y.-D., Liu, Y., Su, J.-T., et al. 2013, Sol. Phys., 288, 585

Shen, Y., Liu, Y., Liu, Y. D., et al. 2015, ApJ, 814, L17

Shen, Y., Liu, Y., Tian, Z., \& Qu, Z. 2017, ApJ, 851, 101

Shen, Y., Liu, Y., Song, T., \& Tian, Z. 2018, ApJ, 853, 1

Sturrock, P. A. 1966, Nature, 211, 695

Su, Y., van Ballegooijen, A., Schmieder, B., et al. 2009, ApJ, 704, 341

Su, Y., Surges, V., van Ballegooijen, A., DeLuca, E., \& Golub, L. 2011, ApJ, 734,53

Su, J. T., Shen, Y. D., \& Liu, Y. 2012, ApJ, 754, 43

Su, W., Cheng, X., Ding, M. D., et al. 2016, ApJ, 830, 70

Tan, B., \& Tan, C. 2012, ApJ, 749, 28

Tian, H. 2017, Res. Astron. Astrophys., 17, 110

Tian, H., \& Chen, N.-H. 2018, ApJ, 856, 34

Tian, H., McIntosh, S. W., \& De Pontieu, B. 2011, ApJ, 727, L37

Tian, H., McIntosh, S. W., Wang, T., et al. 2012, ApJ, 759, 144

Tian, H., DeLuca, E., Reeves, K. K., et al. 2014, ApJ, 786, 137

Tian, H., Young, P. R., Reeves, K. K., et al. 2015, ApJ, 811, 139

Tian, H., Young, P. R., Reeves, K. K., et al. 2016, ApJ, 823, L16

Torrence, C., \& Compo, G. P. 1998, Bull. Am. Meteorol. Soc., 79, 61

Uchida, Y. 1970, PASJ, 22, 341

van Ballegooijen, A. A. 2004, ApJ, 612, 519

van Ballegooijen, A. A., Priest, E. R., \& Mackay, D. H. 2000, ApJ, 539, 983

Van Doorsselaere, T., Kupriyanova, E. G., \& Yuan, D. 2016, Sol. Phys., 291, 3143

Verth, G., \& Erdélyi, R. 2008, A\&A, 486, 1015

Verwichte, E., \& Kohutova, P. 2017, A\&A, 601, L2

Wang, T., Solanki, S. K., Curdt, W., Innes, D. E., \& Dammasch, I. E. 2002, ApJ, 574, L101

Wang, T., Ofman, L., Davila, J. M., \& Su, Y. 2012, ApJ, 751, L27

Wang, T. J., Ofman, L., \& Davila, J. M. 2009, ApJ, 696, 1448

Yang, S., \& Xiang, Y. 2016, ApJ, 819, L24

Yang, W. H., Sturrock, P. A., \& Antiochos, S. K. 1986, ApJ, 309, 383

Young, P. R., Tian, H., \& Jaeggli, S. 2015, ApJ, 799, 218

Yuan, D., \& Van Doorsselaere, T. 2016a, ApJS, 223, 23

Yuan, D., \& Van Doorsselaere, T. 2016b, ApJS, 223, 24

Yuan, D., Nakariakov, V. M., Chorley, N., \& Foullon, C. 2011, A\&A, 533, A116

Yuan, D., Su, J., Jiao, F., \& Walsh, R. W. 2016, ApJS, 224, 30

Zhang, Q. M., \& Ji, H. S. 2014, A\&A, 567, A11

Zhang, Q. M., Li, D., \& Ning, Z. J. 2016, ApJ, 832, 65

Zimovets, I. V., \& Struminsky, A. B. 2010, Sol. Phys., 263, 163

Zimovets, I. V., \& Nakariakov, V. M. 2015, A\&A, 577, A4

Zucca, P., Carley, E. P., Bloomfield, D. S., \& Gallagher, P. T. 2014, A\&A, 564, A47 